\title{
Differential roles of Src in transforming growth factor-ß regulation of growth arrest, epithelial-to-mesenchymal transition and cell migration in pancreatic ductal adenocarcinoma cells
}

\author{
HENDRIK UNGEFROREN ${ }^{1,2}$, SUSANNE SEBENS ${ }^{3,4}$, STEPHANIE GROTH ${ }^{1,5}$, \\ FRANK GIESELER $^{2}$ and FRED FÄNDRICH ${ }^{1}$ \\ ${ }^{1}$ Clinic for Applied Cellular Medicine, UKSH, Campus Kiel, D-24105 Kiel; ${ }^{2}$ First Department of Medicine, UKSH, \\ Campus Lübeck, D-23538 Lübeck; ${ }^{3}$ Institute of Experimental Medicine, Division of Inflammation associated carcinogenesis, \\ Christian-Albrechts-University Kiel; ${ }^{4}$ c/o Laboratory of Molecular Gastroenterology and Hepatology, \\ Department of Internal Medicine I, UKSH, Campus Kiel, D-24105 Kiel, Germany
}

Received October 8, 2010; Accepted December 13, 2010

DOI: $10.3892 /$ ijo.2011.897

\begin{abstract}
Both transforming growth factor (TGF)- $\beta$ and the non-receptor tyrosine kinase Src play major roles during tumorigenesis by regulating cell growth, epithelial-to-mesenchymal transition (EMT), migration/invasion and metastasis, but little is known about the signaling crosstalk between them. To interfere with Src function in vitro and in vivo many studies have employed the pharmacologic Src inhibitors PP2 and PP1. Both agents have recently been shown to be powerful inhibitors of TGF- $B$ receptor type I/ALK5 and type II. As this situation prohibited any definite conclusions with respect to the relative contribution of TGF- $\beta$ vs. Src signaling, we decided to reappraise a potential role of Src in TGF-B1-mediated cellular responses using RNA and dominant-negative (dn) interference to block Src expression and function, respectively. In TGF- $\beta$-responsive pancreatic ductal adenocarcinoma (PDAC) cells, we show that Src is activated by TGF- $\beta 1$ and that its specific inhibition strongly attenuated basal proliferation
\end{abstract}

Correspondence to: Professor Hendrik Ungefroren, First Department of Medicine, UKSH, Campus Lübeck, D-23538 Lübeck, Germany

E-mail: hendrik.ungefroren@uk-sh.de

Present address: ${ }^{5}$ Department of Dermatology, UKSH, Campus Lübeck, D-23538 Lübeck, Germany

Abbreviations: ALK5, activin receptor-like kinase 5; ca, constitutively active; CRNK, calcium regulated non-kinase; EMT, epithelialto-mesenchymal transition; ERK, extracellular signal-regulated kinase; FCS, fetal calf serum; FRNK, FAK-related non-kinase; IP, immunoprecipitation; kd, kinase-deficient; MAPK, mitogenactivated protein kinase; PAI-1, plasminogen activator inhibitor-1; PDAC, pancreatic ductal adenocarcinoma; SFK, Src family kinase; TBP, TATA box binding protein, TGF- $\beta$, transforming growth factor- $\beta$

Key words: Src, TGF- $\beta$, Smad, p38 MAPK and enhanced TGF-ß1-mediated growth arrest. However, Src inhibition was unable to impair TGF-ß1-controlled EMT as evidenced by cell morphology and regulation of the epithelial marker E-cadherin. Despite its dispensibility for TGF-ß-induced EMT, specific inhibition of Src dramatically reduced basal and TGF-B1-induced cell migration in Panc-1 cells as measured with a novel real-time migration assay (xCELLigence DP system). Biochemically, dnSrc inhibition failed to block TGF-31/ALK5-induced activation of Smad2 and Smad3, but partially inhibited transcriptional activation of TGF- $\beta /$ Smad-responsive reporter genes, and effectively blocked basal and TGF- $\beta 1$-induced activation of p38 MAPK. Together, the data provide evidence for a role of Src in the regulation of basal proliferation as well as in basal and TGFß1-mediated cell motility but not EMT in TGF-ß-responsive pancreatic (tumor) cells.

\section{Introduction}

Src kinases are non-receptor intracellular tyrosine kinases that mediate a variety of cellular responses. The prototype member $\mathrm{p} 60^{\mathrm{Src}}$ is the product of the proto-oncogene $c-\mathrm{Src}$, and is the cellular homolog of the Rous sarcoma virus transforming protein $\mathrm{v}-\mathrm{Src}$. Src kinases are involved in numerous signaling pathways controlling proliferation, migration, adhesion, and angiogenesis (1). Src together with $\alpha_{v} \beta 3$ integrin promotes anchorage-independent growth and metastasis (2) and recent data have revealed that transient activation of Src by immortalized mammary epithelial cells triggered an inflammatory response that resulted in the initiation and maintenance of cellular transformation and a switch from a non-transformed to a transformed phenotype (3). Src mediates signaling from many types of receptors including receptor tyrosine kinases (EGF-R), integrins, and G-protein-coupled receptors such as proteinase-activated receptors (PARs) (4). Src is required for EGF-R stimulated DNA synthesis and has been reported to stimulate IGF-I-dependent cell proliferation by increasing IGF-I receptor number (5). Moreover, overexpression of activated c-Src has been reported to stimulate 
migration, downregulation of E-cadherin (6), and an increase in IL-8 expression and angiogenesis (7). Pharmacologic Src inhibition demonstrated significant antitumor and antimetastatic activity in an orthotopic mouse model of human PDAC (8) and reverted inherent and acquired chemoresistance in pancreatic tumor cells in vitro $(9,10)$ and in vivo $(10)$.

Transforming growth factor (TGF-) $B 1$ is a pleiotropic growth factor that controls proliferation, cell migration, and morphological plasticity such as epithelial-to-mesenchymal transition (EMT). TGF- $\beta$ signals through two membranebound receptors designated type II (TßRII) and type I/ALK5. After being phosphorylated by TBRII on serine/threonine residues, ALK5 activates the canonical Smad pathway by phosphorylating receptor-regulated Smads (R-Smads) Smad2 and Smad3. Activated R-Smads subsequently form a complex with Smad4 (also known as DPC4) which moves into the nucleus to regulate the transcriptional activity of TGF- $\beta$ sensitive target genes (11). Besides Smads, other signaling pathways can be activated by the ALK5 kinase including p38 mitogen activated protein kinase (MAPK), which in turn can occur in a Smad-dependent or Smad-independent manner (12). Due to its potent antiproliferative effects on epithelial cells, TGF- $\beta$ is generally viewed as a tumor suppressor.

Pancreatic ductal adenocarcinoma (PDAC) is a deadly disease in which nonsurgical therapy is ineffective and in which the majority of patients harbor metastatic lesions at presentation, precluding the possibility for curative surgical intervention (13). Treatment of the primary tumor is hampered by its high therapy chemoresistance, the immunosuppressive environment, and a stroma-rich desmoplastic reaction. At the molecular level PDAC is characterized by the presence of mutations in the TGF- $\beta$ pathway (40-50\% for Smad4, 3-10\% for $T G F B R I I)$ as well as frequent overexpression of all three isoforms of TGF-ß (14) and Src (15). In a mouse model of human pancreatic cancer, inactivating mutations in the genes for Smad4 or TßRII in pancreatic duct epithelial cells eventually result in the formation of PDAC $(16,17)$. Later in pancreatic tumorigenesis, however, TGF- 3 through ALK5 may promote chemoresistance, migration, angiogenesis, tumor cell invasion, and eventually metastasis $(8,18-21)$, explaining its dual role in carcinogenesis through noncanonical TGF- $\beta$ signaling pathways such as $\mathrm{p} 38$.

Given their overlapping spectra of cellular activities, their causative role in initiation and maintenance of cellular transformation as well as their oncogenic effects during later stages of tumorigenesis, it is not surprising that there is evidence for signaling crosstalk between Src and TGF-3/ ALK5, e.g., in breast cancer cells $(20,22)$. However, whether Src is directly involved in TGF- $\beta$-induced growth control, EMT, and cell migration in PDAC cells has not yet been analysed. This knowledge is crucial to help deciding whether it is feasable to therapeutically target Src and TGF- $ß$ signaling individually or in a combined approach. Previous studies that attempted to inhibit Src's oncogenic functions in vivo often employed the common Src family kinase (SFK) inhibitors PP2 and PP1 $(9,23,24)$. However, we (unpublished data) and others (25) have recently identified PP2 and PP1, respectively, as potent inhibitors of the kinase activity of TßRI and II, complicating the interpretation of in vivo and in vitro data obtained with these agents and raising the possibility that cellular effects ascribed to Src were in fact mediated in part or exclusively by the TGF- $\beta$ receptor(s). Consequently, in order to test whether Src and TGF- $\beta$ signaling indeed cooperate and to distinguish effects of Src from those of TGF- $\beta$ signaling it is mandatory that specific (genetic) inhibition strategies be used. Here, we have used dominant negative (dn), or RNA interference (RNAi), in combination with the relatively specific Src inhibitor SU6656 (26) to suppress Src activity and expression, respectively. We reveal that Src promotes basal growth and antagonizes TGF-ß1-induced growth inhibition while it does not appear to be involved in TGF-ß1-induced EMT in either cell line.

\section{Materials and methods}

Antibodies and reagents. The antibodies and their suppliers were: phospho-Src (Tyr418): Biosource (Nivelles, Belgium), v-Src (Ab-1): Merck Biosciences (Darmstadt, Germany), Src (GD11): Upstate Biotechnology (via Sigma, Deisenhofen, Germany), p125 $5^{\text {FAK }}$ (focal adhesion kinase, FAK), Smad2/3 (E-20), and HSP90: Santa Cruz Biotechnology (Heidelberg, Germany), phospho-Smad2 (Ser465/467), phospho-Smad3 (Ser423/425)/Smad1(Ser463/465), phospho-p38 (Thr180/ Tyr182), and p38: Cell Signaling Technology (via New England Biolabs, Frankfurt, Germany), Smad2: Zymed (Berlin, Germany), B-actin: Sigma. SU6656 was purchased from Merck Biosciences, and SB431542 from Tocris (Ellisville, MO). Pharmacological inhibitors were added to cells 30-60 min before the addition of TGF-B1 (R\&D Systems, Wiesbaden, Germany) which was used at a concentration of $5 \mathrm{ng} / \mathrm{ml}$. All other reagents used were of analytical grade purity.

Cell culture. Human pancreatic adenocarcinoma Panc-1 and Colo 357 cells were maintained as described earlier (27). The generation of the HPDE6c7 cell line (a kind gift of M.S. Tsao) is described elsewhere $(28,29)$. These cells were cultured in RPMI-1640 medium and K-SFM (PAA Laboratories, Cölbe, Germany) (1:1) supplemented with $0.5 \%$ L-glutamine, $50 \mu \mathrm{g} / \mathrm{ml}$ bovine pituitary extract and $5 \mathrm{ng} / \mathrm{ml}$ EGF (Invitrogen, Karlsruhe, Germany), 5\% fetal calf serum (FCS), $100 \mu \mathrm{g} / \mathrm{ml}$ penicillin/streptomycin (both from Biochrom KG, Berlin, Germany). Panc-1 cells stably transduced with retroviral vector were cultured in the presence of $700 \mu \mathrm{g} / \mathrm{ml}$ geneticin (Invitrogen).

RNA isolation and quantitative real-time RT-PCR analysis. Total RNA was isolated with PeqGoldRNApure (peqlab, Erlangen, Germany) and reverse-transcribed using SuperScript II reverse transcriptase (Invitrogen). The conditions for quantitative real-time PCR (qPCR) as well as PCR oligonucleotide primer sequences for $\beta$-actin, TATA-box binding protein (TBP) and GAPDH (30), E-cadherin and Snail2/Slug (31) were described in detail earlier. Real-time PCR was performed on an I-cycler with IQ software (Bio-Rad, München, Germany). All values for E-cadherin and Slug were normalized to those for at least two different housekeeping genes in the same sample to account for small differences in cDNA input.

Construction of vectors and retroviral infection. A cDNA for dn murine Src (Y416F, K295R, in pUSEamp) was purchased 
from Upstate Biotechnology (via Biomol, Hamburg, Germany). For retroviral expression of $\mathrm{dnSrc}$, the entire open reading frame was excised with $C l a \mathrm{I}$ and PmeI and inserted into the retroviral vector TJBA5bMoLink-neo [TJ-neo (27)]. Positive clones (evaluated by PCR, restriction analysis and sequencing of the plasmid-cDNA junctions) were cotransfected into $293 \mathrm{~T}$ producer cells along with retroviral packaging vectors as described previously (27). Retroviral particles released by 293T cells were used to infect Panc-1 cells. Pools and individual clones of productively infected cells were obtained after selection with geneticin. The dnSrc cDNA was also cloned into pcDNA3 for use in transient transfections. Expression vectors for dn versions of FAK (FRNK, FAK-related nonkinase) and Pyk2 (CRNK, calcium regulated non-kinase) were generated by cDNA amplification with primers FRNK-for (5'-catgaggatggagtccagaagac-3') and FRNK-rev (5'-tcagtgtggt ctcgtctgcc-3'), and CRNK-for (5'-taccatggagaaggacattgc catggag-3') and CRNK-rev (5'-cgtcactctgcaggtgggtg-3'), respectively, and $P f u$ polymerase (Stratagene, Heidelberg, Germany). The resulting fragments were subcloned in sense orientation into the EcoRV site of pcDNA3 and verified by restriction enzyme analysis and sequencing.

Transient transfections and reporter gene assays. Small interfering (si)RNA to Src (Src siRNA kit, Dharmacon via Biomol) was transfected twice (on two consecutive days) serum-free into Panc-1 cells with either Lipofectamine 2000 or Lipofectamine RNAiMAX (both from Invitrogen) according to the manufacturer's instructions. Transfected cells were subjected to cell lysis (for immunoblot analysis, see below), $\left[{ }^{3} \mathrm{H}\right]$-thymidine incorporation assays, cell migration assays, see below), or reporter gene assays. For the latter, Panc-1 cells were seeded in 96-well plates and were cotransfected on the next day serum-free with Lipofectamine 2000 with various cDNAs (in pcDNA3) at an equal molar ratio together with 3TPlux (kindly provided by J. Massague), or p6SBE-luc (kindly provided by S.E. Kern) and the Renilla luciferase encoding vector pRL-TK (Promega, Heidelberg, Germany). Each well received the same total amount of DNA. Following transfection luciferase activities were measured with the Dual Luciferase Assay System (Promega). In the reporter gene assays the data were derived from 6-wells processed in parallel and corrected for transfection efficiency with Renilla luciferase activity.

Real-time measurement of cell migration. Using the xCELLigence RTCA DP device from Roche Diagnostics (Mannheim, Germany) real-time measurements of cell migration on wild-type or transfected Panc-1 and Colo 357 cells were performed. For this purpose, 60,000-90,000 cells were seeded per well in CIM-Plates 16 (Roche Diagnostics). Prior to cell seeding the underside of the wells from the upper chamber was coated with $30 \mu \mathrm{l}$ of collagen I (Sigma, Deisenhofen, Germany). Collagen I (400 $\mu \mathrm{g} / \mathrm{ml})$ was chosen since it represents the major matrix protein in PDAC tissue. Growth factors (TGF-ß31, EGF) and inhibitors (SB431542, SU6656) were dissolved in culture medium containing $1 \%$ FCS added to both lower and upper wells at the same concentration. The assay was performed as detailled by Roche Diagnostics in the instruction manual. In those experiments in which cells underwent transfection they were processed to enter the assay 24-48 $\mathrm{h}$ after the second round of transfection. Data acquisition and analysis was performed with the RTCA software (version 1.2, Roche Diagnostics).

Immunoblot analysis and immunoprecipitation. For immunoblot analysis cells were lysed immediately after treatment in either Laemmli buffer or RIPA buffer and the protein concentration was determined with the Bradford assay (BioRad) followed by SDS-PAGE and transfer of fractionated proteins to PVDF membrane. Endogenous Src protein was immunoprecipitated from Panc-1 cellular lysates with antiv-Src (Ab-1) and protein G Plus Sepharose (Santa Cruz Biotechnology) according to the protocol provided by the supplier and subsequently analyzed by immunoblotting with an anti-Src antibody (clone GD11).

$\left[{ }^{3} \mathrm{H}\right]$-thymidine incorporation assay. Forty-eight hours after transfection (see above) cells were trypsinized, counted and seeded in 96-well plates. The next day cells were treated with TGF- $\beta 1$ in normal growth medium for $48 \mathrm{~h}$ and either labeled with [methyl- $\left.{ }^{3} \mathrm{H}\right]$-thymidine for the last $4 \mathrm{~h}$ of the incubation period. $\left[{ }^{3} \mathrm{H}\right]$-thymidine-labeled cells were lysed and counted in a $\beta$-counter as described in detail previously (32) Assays were run in the 96-well format with 6-wells processed in parallel for all treatments and controls.

Statistical analysis. Statistical significance was calculated using the unpaired Student's t-test. Data were considered significant at $\mathrm{p}<0.05$.

\section{Results}

TGF- $\beta 1$ stimulation of Panc- 1 cells activates $c-S r c$. PDAC tissues have been reported to overexpress Src relative to normal pancreatic tissue (15). To confirm that this is reproduced also at the tumor cell level, we monitored expression of total Src in a series of established PDAC cell lines and in the nontumorigenic human pancreatic ductal epithelial cell line HPDE6c7. Panc-1 and Colo 357, two PDAC cell lines known to have retained TGF- $\beta$ responsiveness, as well as HPDE6c7 cells all exhibited moderate to high Src protein levels as determined by immunoblot analysis (Fig. 1A). All further experimentation on malignant cells was done primarily with Panc-1 cells since a large number of studies on the role of both TGF- $\beta$ and Src $(5,7,9,19)$ in PDAC tumorigenesis has been performed with this cell line. Next, we tested whether Src can be activated by TGF- $\beta 1$ in Panc- 1 cells. For this purpose, we determined phosphorylation at Y418, the major autophosphorylation site and marker of Src kinase activation, after various lengths of TGF- $\$ 1$ treatment. Src phosphorylation at this site increased in a time-dependent albeit delayed manner peaking at $4 \mathrm{~h}$ post TGF- $\beta 1$ addition (Fig. 1B). This indicates that Src is expressed in Panc-1 cells and is susceptible to activation by exogenous TGF- $\beta 1$.

Dn Src and Src siRNA both suppress basal proliferation and enhance TGF- $\beta 1$-mediated growth arrest. To confirm the role of Src in modifying TGF- 31 -induced growth responses, we generated Panc-1 cells stably expressing dn Src (Y416F, 
A

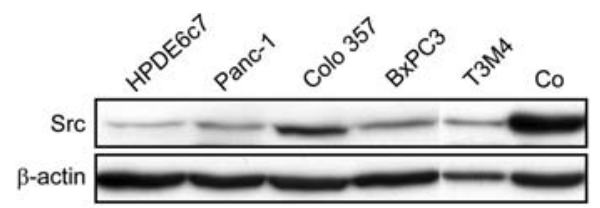

B

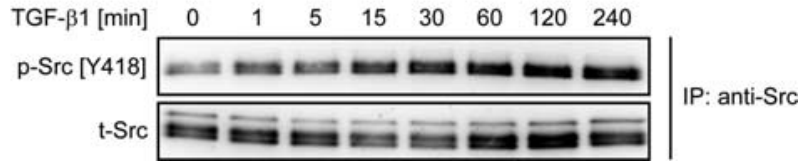

Figure 1. Expression of $\mathrm{c}-\mathrm{Src}$ and its activation by TGF- $\beta 1$ in pancreatic cells. (A) Immunoblot analysis of c-Src (Src) expression in HPDE6c7 cells and various PDAC cell lines. Cells were grown to subconfluency in normal growth medium, harvested and cell lysates collected. Blots were probed with anti-Src and an anti- $\beta$-actin antibody to control for equal loading. Co, control; Panc-1 cells stably transfected with an expression vector (pcDNA3) containing a dn Src cDNA. (B) Kinetics of TGF-ß1-induced c-Src phosphorylation on Tyr-418. Panc-1 cells were cultured as in (A) then serum starved $(0.5 \%$ FCS $)$ for $24 \mathrm{~h}$ and stimulated with TGF- 31 for various times as indicated. C-Src activation was determined by immunoprecipitation (IP) of total c-Src and subsequent immunoblot analysis with anti-phospho-Src [p-Src (Y418)]. The blot was stripped and reprobed for total c-Src (t-Src).
K295R) from a retroviral vector. After selection with geneticin individual clones were shown by immunoblotting to express the Src mutant (Fig. 2A). When tested in a $\left[{ }^{3} \mathrm{H}\right]$-thymidine incorporation assay these clones were found to exhibit strong growth inhibition in both untreated and TGF-B1-treated cells (Fig. 2B). Very similar results were obtained when Panc-1 cells (Fig. 2C) or non-tumorigenic pancreatic ductal epithelial HPDE6c7 cells (Fig. 2D) were first transiently transfected with siRNA to Src and subsequently subjected to DNA synthesis measurements in the absence and presence of TGF- $\beta 1$. In addition, in Colo 357 cells exposed to SU6656 both basal and TGF-B1-inhibited DNA synthesis was further reduced (data not shown). The data clearly show that specific inhibition of Src in PDAC cells by lowering its activity (dn Src, SU6656) or expression (siRNA) strongly reduces basal growth and enhances TGF- $\beta 1$-induced growth inhibition. We further conclude that these effects of Src were not confined to PDAC cells since they also operate in premalignant HPDE6c7 cells.

Inhibition of Src fails to block TGF- $\beta 1$-induced EMT. Panc-1 cells have been shown to undergo TGF-ß1-induced EMT
A

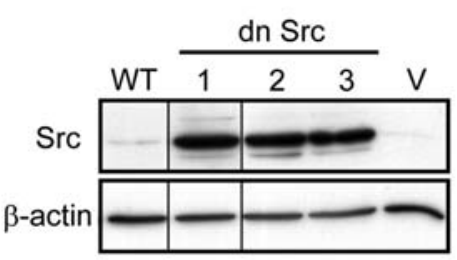

B

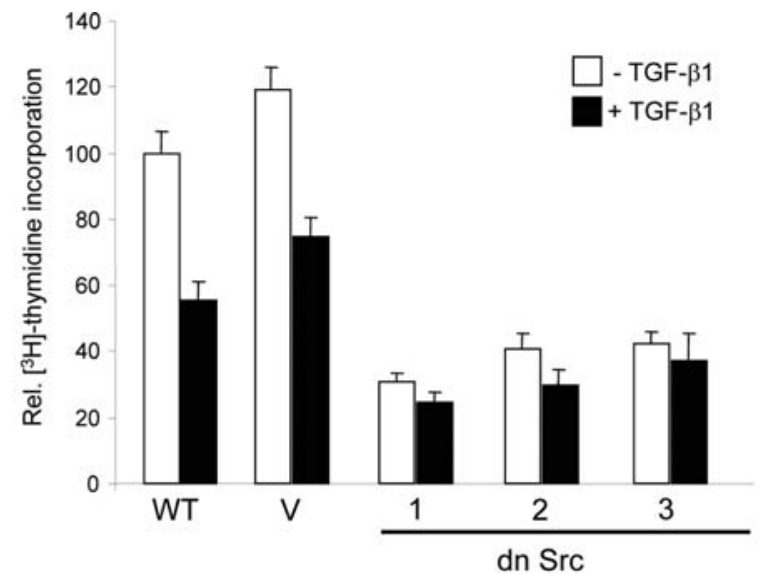

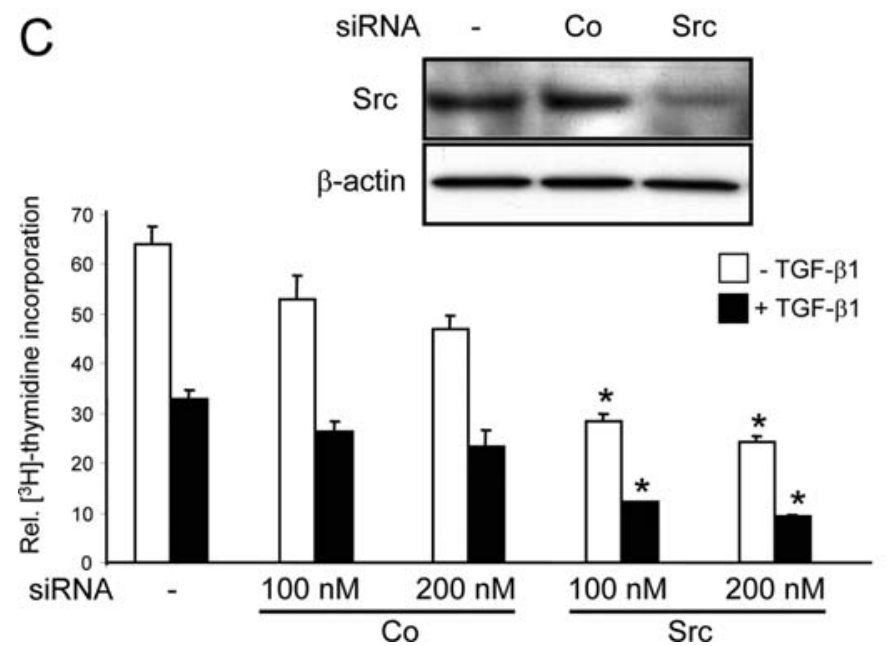

D

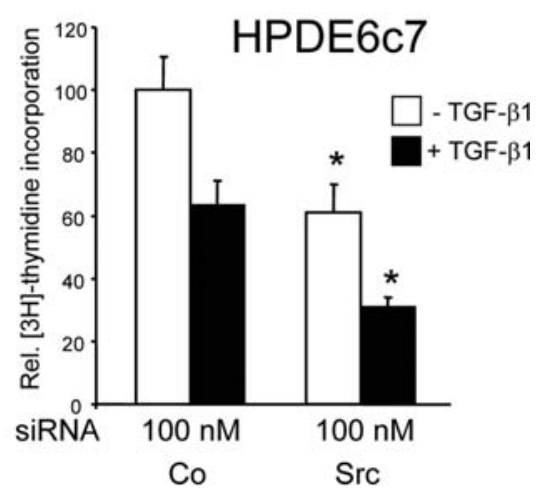

Figure 2. Specific inhibition of c-Src suppresses basal proliferation and enhances TGF-ß1-mediated growth arrest in Panc-1 cells. (A) Western blot analysis of c-Src expression in three individual Panc-1 cell clones derived from stable transduction with dn Src-encoding retrovirus or empty retrovirus (V). WT, wild-type cells. (B) $\left[{ }^{3} \mathrm{H}\right]$-thymidine incorporation assay of individual Panc-1-dn Src clones and controls cultured in normal growth medium in the absence or presence of TGF-B1 for 24 h. (C) [ $\left.{ }^{3} \mathrm{H}\right]$-thymidine incorporation assay of Panc-1 cells treated with lipofectamine alone (-) or transfected with control (Co) siRNA or Srcspecific siRNA (each at a concentration of 100 and $200 \mathrm{nM}$ ) by lipofection. Forty-eight hours after the start of transfection cells were stimulated with TGF- $\beta 1$ for $24 \mathrm{~h}$ and subjected to $\left[{ }^{3} \mathrm{H}\right]$-thymidine labeling. The inset shows Src immunoblotting of Src siRNA transfected Panc-1 cells. (D) As in (C) except that HPDE6c7 cells were transfected with $100 \mathrm{nM}$ of Co and Src-specific siRNAs. Data in (B-D) were derived from 3 independent experiments and represent the means $\pm \mathrm{SD} ;{ }^{*} \mathrm{p}<0.05$, vs. the respective Co siRNA of the same concentration and treatment. 
A

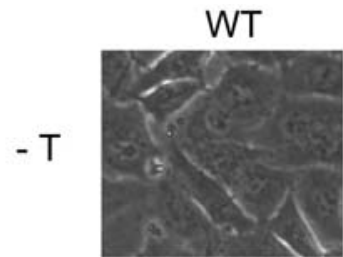

B

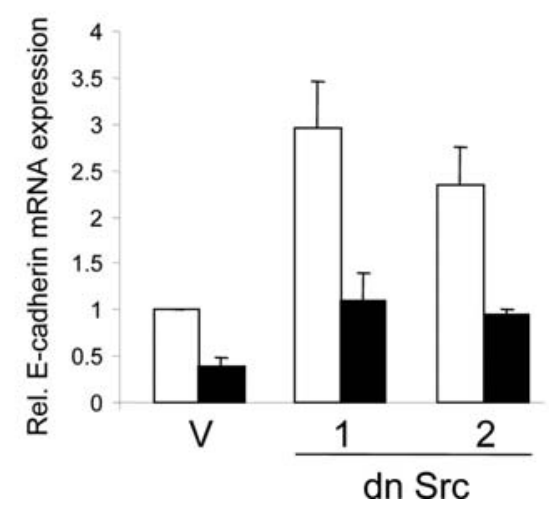

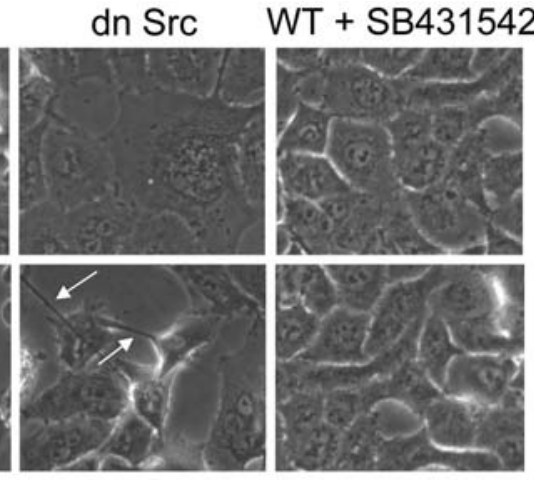

Slug

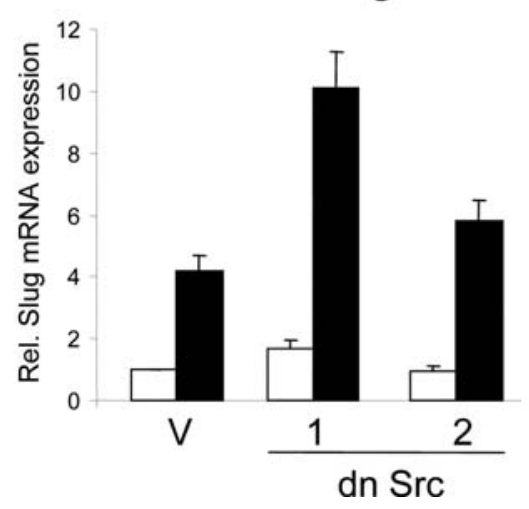

Figure 3. Effects of ectopic expression of dn Src on TGF-ß1-induced EMT. (A) Wild-type (WT) Panc-1 cells and Panc-1 cells expressing empty vector (V) or dn Src remained unstimulated (- T) or were treated with $5 \mathrm{ng} / \mathrm{ml}$ TGF- $\beta 1(+\mathrm{T})$ for $48 \mathrm{~h}$. As control, WT cells were treated in parallel with TGF- 1 in the presence of the ALK5 inhibitor SB431542 (1 $\mu \mathrm{M})$. Original magnification of each photomicrograph x200. (B) Panc-1-empty vector and dn Src expressing cells were challenged with TGF- $\$ 1$ for $48 \mathrm{~h}$ followed by RNA isolation and qPCR for E-cadherin and Slug. Data shown represent the mean values \pm SD normalized to GAPDH and TBP and are representative of three individual experiments all with very similar results.

$(33,34)$ which has been associated with an invasive phenotype and migratory behavior (33). To evaluate whether Src was involved in mediating EMT in PDAC cells, we utilized the Panc-1 cells ectopically overexpressing dn Src. Parental Panc- 1 cell cultures treated for $48 \mathrm{~h}$ with TGF- $\beta 1$ presented with a fibroblastoid morphology and long slender processes (Fig. 3A, arrows), whereas untreated cells were tightly packed and exhibited a cobblestone-like growth pattern. Noteworthy, Panc-1-dn Src cells failed to suppress these morphologic changes upon TGF-ß1 stimulation (Fig. 3A). Similar data were obtained upon SU6656-mediated Src inhibition in Colo 357 cells (data not shown). We also analysed in TGF-ß1treated dn Src-expressing and empty vector control Panc-1 cells the EMT marker E-cadherin the transcriptional silencing of which represents a hallmark and causative event in TGFß1-induced EMT, and the EMT-associated transcription factor Slug. As expected from the morphology data, stable transfection with dn Src was unable to prevent the TGF-ß1induced downregulation of E-cadherin and upregulation of Slug as measured by qPCR (Fig. 3B). Rather, dn inhibition of Src even enhanced both basal and TGF-ß31-controlled E-cadherin mRNA expression (without releaving the inhibitory TGF- 3 effect), consistent with an earlier report that activated Src downregulates E-cadherin gene expression in pancreatic cancer cell lines (6). Interestingly, dn Src also potentiated
TGF-B1-induced Slug expression (Fig. 3B). Together, the data illustrate that Src is dispensable for TGF- $\beta 1$-induced EMT in PDAC cells.

Specific inhibition of Src suppresses both basal and TGF- $\beta 1$ induced cell migration. Prompted by the finding that inhibition of Src was unable to prevent the morphologic and molecular changes associated with TGF-ß1-induced EMT, we asked whether Src would be involved in controlling basal and TGFß1-induced cell motility. All cell migration data were acquired with the xCELLigence RTCA DP device, a novel real-timebased cell migration assay which allows for continuous data recording over a period of several days. Moreover, in contrast to endpoint assays like the standard transwell assay this device allows to precisely determine the peak of migratory activity of a given cell population in culture. Panc-1 cells responded to TGF- $\beta 1$ stimulation with strongly enhanced cellular migration as determined with the xCELLigence assay (Fig. 4). Measurement of untreated and TGF-B1-stimulated Panc-1-dn Src cells (two individual clones) showed that both basal and TGF- 31 -triggered migratory activity was dramatically reduced (Fig. 4A). Very similar results were obtained following transient transfection of Panc-1 cells with Src siRNA (Fig. 4B). The migratory response to exogenous TGF-ß1 was ALK5-dependent as concomitant treatment 
A
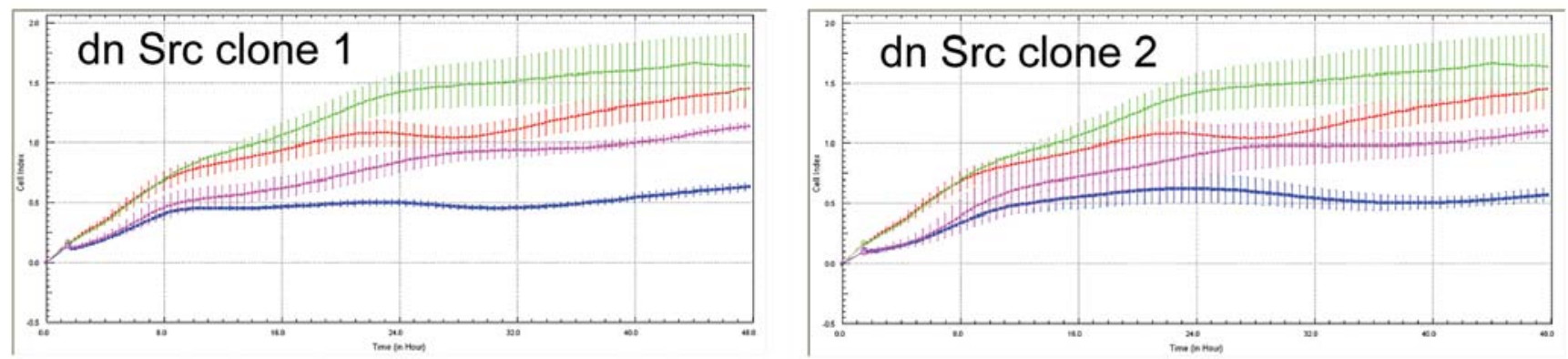

B

C
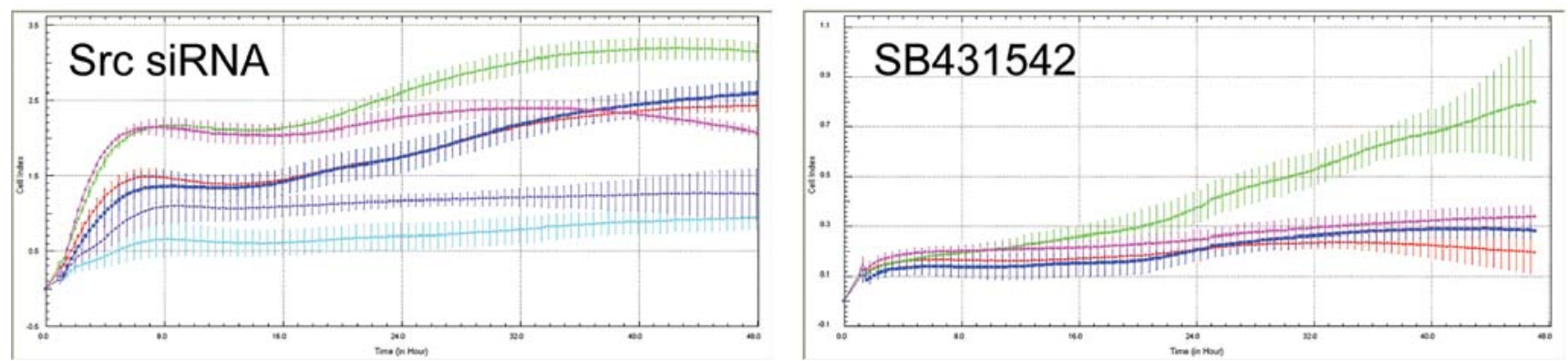

Figure 4. Effect of Src inhibition on TGF-ß1-induced cell migration of Panc-1 cells as measured with a novel real-time cell migration assay. (A) Effect of stable ectopic expression of dn Src. Two individual clones of Panc-1 cells ectopically overexpressing dn Src (Fig. 2A) were resuspended in medium with $1 \%$ FCS and seeded into the wells of a CIM-Plate $16(60,000 /$ well $)$ of the RTCA DP instrument. Cells were allowed to migrate in the presence of TGF- 11 ( $5 \mathrm{ng} / \mathrm{ml}$, added to both the lower and upper compartment). Changes in impedance resulting from cells that have migrated to the underside of wells (chemokinesis) were recorded every $15 \mathrm{~min}$ and monitored for a total of $48 \mathrm{~h}$. Red curve, untreated vector control cells; green curve, TGF- $\beta 1$-treated vector control cells; blue curve, untreated dn Src expressing cells; pink curve, TGF-ß31-treated dn Src expressing cells. (B) Panc-1 cells transfected twice (on days 2 and 3 after seeding) with control and Src-specific siRNAs were trypsinized on day 4 and subjected to RTCA DP assay as described in (A). Red curve, untreated lipofectamine treated cells; green curve, TGF-B1-treated lipofectamine treated cells; dark blue curve, untreated control siRNA transfected cells; pink curve, TGF-ß1-treated control siRNA transfected cells; turquoise curve, untreated Src siRNA transfected cells; light blue curve, TGF-ß1-treated Src siRNA transfected cells. (C) Effect of the specific ALK5 inhibitor SB431542 on TGF-ß1-induced cell motility. Wild-type Panc-1 cells were processed for the RTCA $\mathrm{DP}$ migration assay as described in (A) and allowed to migrate for $48 \mathrm{~h}$ in the absence or presence of TGF- 31 and SB431542 (1 $\mu \mathrm{M})$. Data in (A-C) were taken from a representative experiment (out of at least three performed in total each with very similar results) and are presented as the mean values \pm SD of quadruplicate wells.

with SB431542 (35) abolished TGF-ß1-induced cell motiliy (Fig. 4C). Together, these results clearly show that although Src is dispensable for TGF- $\beta 1$-induced EMT, it is required for basal and TGF-ß1-induced cell migration in Panc-1 cells.

Src inhibition does not interfere with TGF- $\beta 1$-induced $R$ Smad activation but inhibits basal and TGF- $\beta 1$-induced p38 MAPK phosphorylation in Panc-1 cells. We have shown above that Src inhibition decreased basal and TGF-B1-induced cell motility as well as basal proliferation but enhanced TGF-ß1induced growth arrest. Since both responses require activation of Smad proteins this suggested the possibility that Src signaling interacts with either TGF-B/Smad and/or non-Smad signaling. To determine whether Src signaling is required for TGF- $3 /$ Smad transcriptional responses, Panc-1 cells were transiently transfected with the Smad-responsive reporter plasmids 3TPlux or p6SBE-luc together with dn mutants of either Src, FAK (FRNK) or the related Pyk2 (CRNK) and subjected to reporter gene assays. Interestingly, $\mathrm{dn} \mathrm{Src}$ and FRNK but not CRNK partially suppressed the TGF-B1 effect on both 3TPlux and p6SBE-luc (Fig. 5A).
To test the possibility that Src inhibits the kinase activity of ALK5, we monitored cytosolic R-Smad activation following TGF-ß1 treatment. C-terminal phosphorylation of Smad2 and Smad 3 by TGF- 31 remained unaffected in Panc-1-dn Src stable clones (Fig. 5B) and in Panc-1 cells transiently cotransfected with FLAG-tagged-Smad2 and dn Src (not shown). As additional controls, we again employed SU6656 which, was also unable to block Smad2 phosphorylation, while SB431542 effectively abolished it (Fig. 5C). Total levels of Smad2 and Smad3 always remained unchanged (Fig. 5B and C). We (30) and others (36) have shown previously that TGF- 31 activates the p38 MAPK pathway in Panc-1 cells which is involved in K-ras-mediated cell migration in these cells (37). It was therefore of interest to analyse whether specific Src inhibition affected p38 phosphorylation in these cells. Interestingly, TGF- 31 -mediated phosphorylation of p38 in Panc-1 cells stably expressing dn Src (the same clones as above) was reduced or abolished when compared to empty vector control cells (Fig. 5D). The data indicate that $\mathrm{Src}$ is required for the activation of $\mathrm{p} 38$ by TGF- $\beta 1$ and, in addition, may partially mediate TGF-ß/ALK5/Smad-induced transcriptional effects at some point downstream of R-Smad activation. 


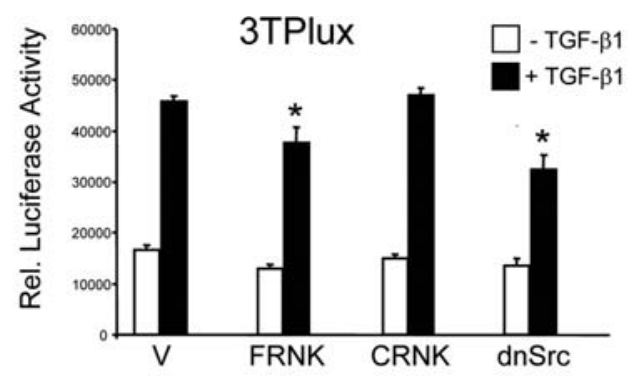

B

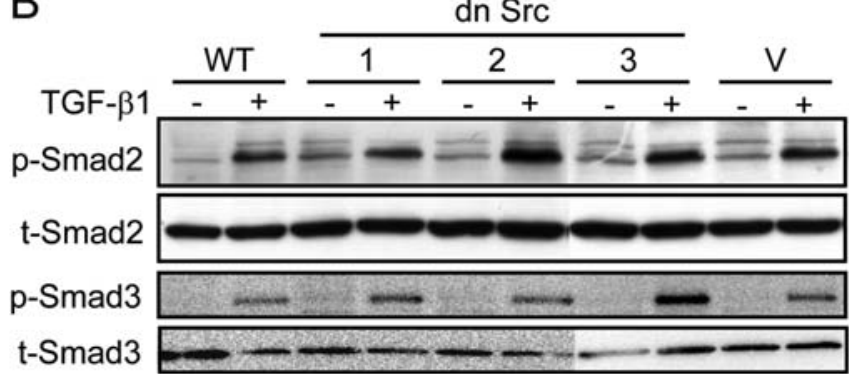

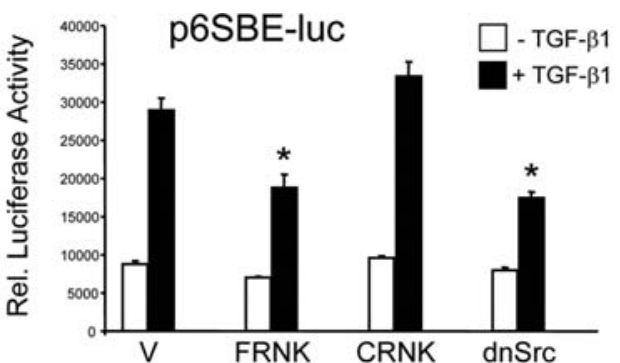

C

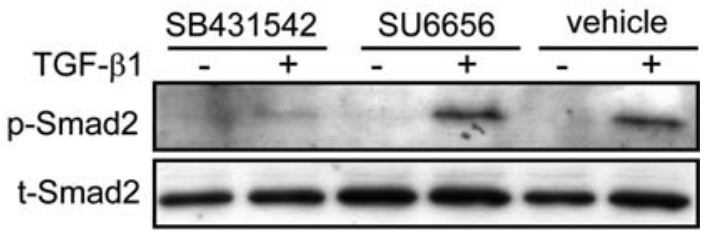

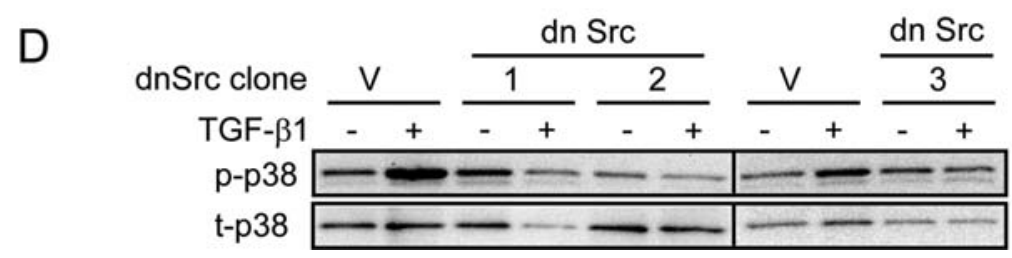

Figure 5. Differential sensitivity of TGF-ß/Smad-responsive reporters to specific inhibition of Src. (A) Panc-1 cells were cotransfected with 3TPlux (left panel) or p6SBE-luc (right panel), together with the Renilla luciferase-encoding vector pRL-TK, and either empty pcDNA3 vector (V), FRNK, CRNK (dn mutants of FAK and Pyk2, respectively), or dn Src for $3 \mathrm{~h}$ using Lipofectamine Plus. Twenty-four hours after the start of transfection, cells were stimulated with TGF- $\$ 1$ for another 24-h period followed by dual luciferase measurements. For all assays, data were from one representative experiment out of 4 done in total and represent firefly luciferase values normalized with those for Renilla luciferase. Data are presented as mean values \pm SD. ${ }^{*}<<0.05$, vs. TGF- $\beta$-treated vector control. (B) Analysis of Smad2 and Smad3 activation by TGF- 31 in Panc-1-dn Src cells. Three different clones of Panc-1-dn Src cells as well as wild-type (WT) and vector (V) controls were treated, or not, for $1 \mathrm{~h}$ with TGF- 31 and evaluated for activation of Smad 2 and Smad3 by immunoblotting for C-terminal phosphorylation of Smad2 and Smad3. The total (t-) R-Smad levels were visualized with antibodies against Smad2 and Smad3. (C) Effect of pharmacologic inhibition of Src on TGF-ß1-induced R-Smad activation. Panc-1 cells were treated for $1 \mathrm{~h}$ with TGF- 31 in the presence of SB431542 (1 $\mu$ M, positive control), the specific Src inhibitor SU6656 $(10 \mu \mathrm{M})$, or vehicle $(0.01 \%$ dimethylsulfoxide) and subjected to measurement of Smad2 phosphorylation as described in (B). (D) Analysis of TGF-B1-induced p38 MAPK activation in Panc-1-dn Src cells. Three different clones of Panc-1-dn Src as well as wild-type (WT) and vector (V) controls were treated, or not, for $1 \mathrm{~h}$ with TGF- 31 and evaluated for activation of p38 by immunoblotting with a phospho-p38 (p-p38) antibody. The total p38 levels were visualized with an antibody to p38 (t-p38).

\section{Discussion}

Both Src and TGF- 3 can drive cancer progression by promoting primary tumor growth, angiogenesis, invasion, migration, and metastasis. In PDAC tissues and cell lines Src and TGF- $\beta 1$ are overexpressed and Src kinase activity is often increased (15). Previous in vitro and in vivo studies that have attempted to block the oncogenic activity of Src have mostly employed pharmacologic inhibitors which generally suffer from a lack of specificity (26). Specifically, PP1 has been shown by Maeda and cowokers (25) to be a powerful inhibitor of TßRI (and TßRII), raising the possibility that effects ascribed to Src were in fact mediated by the TßRs. In this study, we employed tumorigenic Panc-1 (primarily) and Colo 357 cells [two TGF-ß1-responsive cell lines most commonly used as orthotopic xenotransplantation models for preclinical evaluation of anticancer agents in $\operatorname{PDAC}(23,24)]$ in conjunction with highly specific genetic inhibition strategies to probe the role of Src in TGF-ß31-mediated cellular responses relevant for PDAC progression. Using these specific inhibition strategies we demonstrate that Src differentially affects TGF-ß1-induced growth arrest, EMT, and cell migration.

In Panc-1 and non-tumorigenic HPDE6c7 cells both stable ectopic expression of $\mathrm{dn} \mathrm{Src}$ as well as siRNA-mediated knockdown of Src suppressed basal growth which is consistent with the growth-promoting function of Src. Both experimental approaches also increased the cytostatic effect of TGF- $\beta 1$ though this effect was more pronounced upon siRNA-mediated silencing of Src (compare panels B and C in Fig. 2). The reason for this discrepancy is unclear at present but may be related to the higher overall inhibition of the basal proliferative activity by dn Src (probably as a result of its expression in all cells of the culture versus expression of siRNA in only a fraction of cells), or, alternatively, may be explained by a 
non-kinase-related function(s) of Src that is still preserved in the dn mutant. Nevertheless, the data indicate that Src was not directly involved in mediating growth inhibition by TGF-ß1.

TGF- $\beta$ is a well known promoter of EMT but whether Src is involved in this process is still a matter of debate. Since no information in this regard was available for PDAC cells, we analysed whether TGF-ß1-induced EMT was sensitive to inhibition of Src. Ectopic expression of dn Src failed to prevent the morphological and transcriptional changes associated with EMT, suggesting that in PDAC cells Src is dispensable for TGF-ß1-induced EMT. Consistent with this, we have shown that treatment with the Src-specific inhibitor SU6656 had no effect either, while SB431542 and PP1, the latter targeting TßRI and Src, completely prevented the morphological changes associated with TGF-ß1-induced EMT [(25); Ungefroren et al, unpublished data]. Contradictory results exist for normal (non-transformed) murine mammary epithelial NMuMG cells; while Maeda et al (25) demonstrated that Src does not play a role in TGF-ß-induced EMT, Galliher and Schiemann (20) reported that Src promoted it by $B 3$ integrinmediated induction of TßRII tyrosine phosphorylation, and ERK1/2 and p38 MAPK activation. Likewise, Galliher and Schiemann also found a role for Src in TGF- $\beta$-induced EMT of breast cancer cells (20). It remains to be seen whether the involvement of Src in EMT is a species-specific, cell type-specific, or context-dependent event, or whether it is even associated with the stage of malignancy within a given tumor entity.

Both dn Src expression and SU6656 treatment failed to suppress Smad2 and Smad3 activation by TGF- 11 but abolished TGF-ß1-induced activation of p38 MAPK, which suggests a role of Src in the activation of p38 by TGF- 31 . Unlike other cellular functions, dn inhibition of Src and FAK, the latter of which is an Src substrate, moderately albeit significantly reduced TGF- $\beta 1$ transcriptional activity on two TGF- $3 /$ Smad-responsive reporters. Together with the positive regulation of the p6SBE-luc reporter by kinase-active Src (Ungefroren et al, unpublished data), these data indicate that in Panc-1 cells Src is partially involved in transcriptional responses to TGF- $\beta 1$ downstream of $\mathrm{Smad} 2 / 3$ activation.

Although Src was dispensable for TGF-B1-induced EMT in both Panc-1 and Colo 357 cells, it clearly enhanced basal migration in Panc-1 cells as observed previously by others (19) and was strictly required for a full TGF- $\beta 1$-induced migratory response in Panc-1 cells. Src regulation of TGF-ß1-dependent cell motility in PDAC cells may be mediated through $\mathrm{p} 38$ MAPK since p38 is required for TGF- $\beta$-mediated cell migration (38). Moreover, Src has been shown to regulate TGF- 3 stimulation of p38 MAPK during invasion and proliferation of breast cancer cells (39). However, in Panc-1 cells activation of p38 is not required for TGF- $\beta 1$-mediated growth arrest (Ungefroren et al, unpublished data), providing an explanation for the differential effect of Src in TGF- 31 control of cell growth and migration. The slow kinetics of TGF-B1-triggered Src phosphorylation (peak at $4 \mathrm{~h}$ of exposure to TGF- $\beta 1$ ) and cell migration (peak between 30 and $40 \mathrm{~h}$ ), however, suggested that these were indirect effects and that other receptors known to activate Src, e.g., EGF-R act downstream of TßRI/ALK5 (40). Crosstalk of TGF- $\beta$ signaling with EGF-R signaling is indeed known to occur in Panc-1 cells during TGF- $\beta$ control of the cytostatic response (41). Other possible candidates are $\mathrm{G}$ protein-coupled receptors such as PARs (4) as we have obtained preliminary evidence that PAR-2 is required for TGF-ß1/ALK5-mediated cell migration possibly through receptor transactivation.

Taken together, the results of this study establish that in PDAC cells Src promotes basal proliferation as well as basal and TGF- $\beta 1$-induced cell migration while it appears to be dispensable for TGF-ß1-mediated growth arrest and EMT. Our results obtained with the use of specific inhibitors might aid in dissociating oncogenic functions exerted by Src from those of the TßRs, the knowledge of which is mandatory for using pharmacologic Src inhibitors in studies of experimental cancer therapeutics of late-stage PDAC.

\section{Acknowledgements}

We thank H. Albrecht, S. Grammerstorf, D. Leisner, and M. Vollmert for excellent technical assistance and Drs S.A. Courtneidge (La Jolla, CA) and S.E. Kern (Baltimore, MD) for generously providing plasmids. This work was supported in part by the Deutsche Forschungsgemeinschaft (DFG) grants UN 128/1-2 (H.U.) and SE1831/2-1 (S.S.).

\section{References}

1. Summy JM and Gallick GE: Src family kinases in tumor progression and metastasis. Cancer Metastasis Rev 22: 337-358, 2003.

2. Desgrosellier JS, Barnes LA, Shields DJ, Huang M, Lau SK, Prévost N, Tarin D, Shattil SJ and Cheresh DA: An integrin alpha(v)beta(3)-c-Src oncogenic unit promotes anchorageindependence and tumor progression. Nat Med 15: 1163-1169, 2009.

3. Iliopoulos D, Hirsch HA and Struhl K: An epigenetic switch involving NF-kappaB, Lin28, Let-7 MicroRNA, and IL6 links inflammation to cell transformation. Cell 139: 693-706, 2009.

4. Su S, Li Y, Luo Y, Sheng Y, Su Y, Padia RN, Pan ZK, Dong Z and Huang S: Proteinase-activated receptor 2 expression in breast cancer and its role in breast cancer cell migration. Oncogene 28: 3047-3057, 2009.

5. Flossmann-Kast BB, Jehle PM, Hoeflich A, Adler G and Lutz MP: Src stimulates insulin-like growth factor I (IGF-I)dependent cell proliferation by increasing IGF-I receptor number in human pancreatic carcinoma cells. Cancer Res 58: 3551-3554, 1998.

6. Menke A, Philippi C, Vogelmann R, Seidel B, Lutz MP, Adler G and Wedlich D: Down-regulation of E-cadherin gene expression by collagen type I and type III in pancreatic cancer cell lines. Cancer Res 61: 3508-3517, 2001.

7. Trevino JG, Summy JM, Gray MJ, Nilsson MB, Lesslie DP, Baker $\mathrm{CH}$ and Gallick GE: Expression and activity of SRC regulate interleukin-8 expression in pancreatic adenocarcinoma cells: implications for angiogenesis. Cancer Res 65: 7214-7222, 2005.

8. Yezhelyev MV, Koehl G, Guba M, Brabletz T, Jauch KW, Ryan A, Barge A, Green T, Fennell M and Bruns CJ: Inhibition of SRC tyrosine kinase as treatment for human pancreatic cancer growing orthotopically in nude mice. Clin Cancer Res 10: 8028-8036, 2004

9. Duxbury MS, Ito H, Zinner MJ, Ashley SW and Whang EE: Inhibition of SRC tyrosine kinase impairs inherent and acquired gemcitabine resistance in human pancreatic adenocarcinoma cells. Clin Cancer Res 10: 2307-2318, 2004.

10. Ischenko I, Camaj P, Seeliger H, Kleespies A, Guba M, De Toni EN, Schwarz B, Graeb C, Eichhorn ME, Jauch KW and Bruns CJ: Inhibition of Src tyrosine kinase reverts chemoresistance toward 5-fluorouracil in human pancreatic carcinoma cells: an involvement of epidermal growth factor receptor signaling. Oncogene 27: 7212-7222, 2008. 
11. Moustakas A, Pardali K, Gaal A and Heldin CH: Mechanisms of TGF-beta signaling in regulation of cell growth and differentiation. Immunol Lett 82: 85-91, 2002.

12. Derynck R and Zhang YE: Smad-dependent and Smad-independent pathways in TGF- $\beta$ family signalling. Nature 425 : 577-584, 2003.

13. Warshaw AL and Fernández del Castillo C: Pancreatic carcinoma. N Engl J Med 326: 455-465, 1992.

14. Friess H, Yamanaka Y, Büchler M, Ebert M, Beger HG, Gold LI and Korc M: Enhanced expression of transforming growth factor beta isoforms in pancreatic cancer correlates with decreased survival. Gastroenterology 105: 1846-1856, 1993.

15. Lutz MP, Esser IB, Flossmann-Kast BB, Vogelmann R, Luhrs H, Friess H, Buchler MW and Adler G: Overexpression and activation of the tyrosine kinase $\mathrm{Src}$ in human pancreatic carcinoma. Biochem Biophys Res Commun 243: 503-508, 1998.

16. Bardeesy N, Cheng KH, Berger JH, Chu GC, Pahler J, Olson P, Hezel AF, Horner J, Lauwers GY, Hanahan D and De Pinho RA: Smad4 is dispensable for normal pancreas development yet critical in progression and tumor biology of pancreas cancer. Genes Dev 20: 3130-3146, 2006.

17. Ijichi H, Chytil A, Gorska AE, Aakre ME, Fujitani Y, Fujitani S, Wright CV and Moses HL: Aggressive pancreatic ductal adenocarcinoma in mice caused by pancreas-specific blockade of transforming growth factor-beta signaling in cooperation with active Kras expression. Genes Dev 20: 3147-3160, 2006.

18. Schniewind B, Groth S, Sebens Müerköster S, Sipos B, Schäfer H, Kalthoff H, Fändrich F and Ungefroren H: Dissecting the role of TGF-beta type I receptor/ALK5 in pancreatic ductal adenocarcinoma: Smad activation is crucial for both the tumor suppressive and prometastatic function. Oncogene 26: 4850-4862, 2007.

19. Ito H, Gardner-Thorpe, Zinner, MJ, Ashley SW and Whang EE: Inhibition of tyrosine kinase Src suppresses pancreatic cancer invasiveness. Surgery 134: 221-226, 2003.

20. Galliher AJ and Schiemann WP: Beta3 integrin and Src facilitate transforming growth factor-beta mediated induction of epithelial-mesenchymal transition in mammary epithelial cells. Breast Cancer Res 8: R42, 2006.

21. Bierie B and Moses HL: Tumour microenvironment: TGFß: the molecular Jekyll and Hyde of cancer. Nat Rev Cancer 6: 506-520, 2006.

22. Wang SE, Xiang B, Zent R, Quaranta V, Pozzi A and Arteaga CL: Transforming growth factor beta induces clustering of HER2 and integrins by activating Src-focal adhesion kinase and receptor association to the cytoskeleton. Cancer Res 69: 475-482, 2009.

23. Donnini S, Monti M, Castagnini C, Solito R, Botta M, Schenone S, Giachetti A and Ziche M: Pyrazolo-pyrimidinederived c-Src inhibitor reduces angiogenesis and survival of squamous carcinoma cells by suppressing vascular endothelial growth factor production and signaling. Int J Cancer 120: 995-1004, 2007.

24. Hirokawa Y, Levitzki A, Lessene G, Baell J, Xiao Y, Zhu H and Maruta H: Signal therapy of human pancreatic cancer and NF1deficient breast cancer xenograft in mice by a combination of PP1 and GL-2003, anti-PAK1 drugs (Tyr-kinase inhibitors). Cancer Lett 245: 242-251, 2007.

25. Maeda M, Shintani Y, Wheelock MJ and Johnson KR: Src activation is not necessary for transforming growth factor (TGF)-\{-mediated epithelial to mesenchymal transitions (EMT) in mammary epithelial cells. J Biol Chem 281: 59-68, 2006.

26. Bain J, McLauchlan H, Elliott M and Cohen P: The specificities of protein kinase inhibitors: an update. Biochem J 371: 199-204, 2003.

27. Chen WB, Lenschow W, Tiede K, Fischer JW, Kalthoff H and Ungefroren H: Smad4/DPC4-dependent regulation of biglycan gene expression by transforming growth factor-beta in pancreatic tumor cells. J Biol Chem 277: 36118-36128, 2002.
28. Liu N, Furukawa T, Kobari M and Tsao MS: Comparative phenotypic studies of duct epithelial cell lines derived from normal human pancreas and pancreatic carcinoma. Am J Pathol 153: 263-269, 1998.

29. Ouyang H, Mou Lj, Luk C, Liu N, Karaskova J, Squire J and Tsao MS: Immortal human pancreatic duct epithelial cell lines with near normal genotype and phenotype. Am J Pathol 157: 1623-1631, 2000.

30. Groth S, Schulze M, Kalthoff H, Fändrich F and Ungefroren H: Adhesion and Rac1-dependent regulation of biglycan gene expression by transforming growth factor-beta. Evidence for oxidative signaling through NADPH oxidase. J Biol Chem 280: 33190-33199, 2005.

31. Geismann C, Morscheck M, Koch D, Bergmann F, Ungefroren H, Arlt A, Tsao MS, Bachem MG, Altevogt P, Sipos B, Fölsch UR, Schäfer H and Sebens Müerköster S: Up-regulation of L1CAM in pancreatic duct cells is transforming growth factor beta1- and slug-dependent: role in malignant transformation of pancreatic cancer. Cancer Res 69: 4517-45426, 2009.

32. Voss M, Wolff B, Savitskaia N, Ungefroren H, Deppert W, Schmiegel W, Kalthoff $\mathrm{H}$ and Naumann M: TGFbeta-induced growth inhibition involves cell cycle inhibitor p21 and pRb independent from p15 expression. Int J Oncol 14: 93-101, 1999.

33. Ellenrieder V, Hendler SF, Boeck W, Seufferlein T, Menke A, Ruhland C, Adler G and Gress TM: Transforming growth factor betal treatment leads to an epithelial-mesenchymal transdifferentiation of pancreatic cancer cells requiring extracellular signal-regulated kinase 2 activation. Cancer Res 61: 4222-4228, 2001.

34. Ellenrieder V, Hendler SF, Ruhland C, Boeck W, Adler G and Gress TM: TGF-beta-induced invasiveness of pancreatic cancer cells is mediated by matrix metalloproteinase- 2 and the urokinase plasminogen activator system. Int J Cancer 93: 204-211, 2001

35. Inman GJ, Nicolas FJ, Callahan JF, Harling JD, Gaster LM, Reith AD, Laping NJ and Hill CS: SB-431542 is a potent and specific inhibitor of transforming growth factor- $\beta$ superfamily type I receptor-like kinase (ALK) receptors ALK4, ALK5, and ALK7. Mol Pharmacol 62: 65-74, 2002.

36. Takekawa M, Tatebayashi $\mathrm{K}$, Itoh $\mathrm{F}$, Adachi $\mathrm{M}$, Imai $\mathrm{K}$ and Saito H: Smad-dependent GADD45beta expression mediates delayed activation of p38 MAP kinase by TGF-beta. EMBO J 21: 6473-6482, 2002

37. Dreissigacker U, Mueller MS, Unger M, Siegert P, Genze F, Gierschik P and Giehl K: Oncogenic K-Ras down-regulates Rac1 and RhoA activity and enhances migration and invasion of pancreatic carcinoma cells through activation of p38. Cell Signal 18: 1156-1168, 2006.

38. Bakin AV, Rinehart C, Tomlinson AK and Arteaga CL: p38 mitogen-activated protein kinase is required for TGFbeta-mediated fibroblastic transdifferentiation and cell migration. J Cell Sci 115: 3193-3206, 2002

39. Galliher AJ and Schiemann WP: Src phosphorylates Tyr284 in TGF-beta type II receptor and regulates TGF-beta stimulation of p38 MAPK during breast cancer cell proliferation and invasion. Cancer Res 67: 3752-3758, 2007.

40. Lee YH, Kayyali US, Sousa AM, Rajan T, Lechleider RJ and Day RM: Transforming growth factor-beta1 effects on endothelial monolayer permeability involve focal adhesion kinase/Src. Am J Respir Cell Mol Biol 37: 485-493, 2007.

41. Giehl K, Seidel B, Gierschik P, Adler G and Menke A: TGFbetal represses proliferation of pancreatic carcinoma cells which correlates with Smad4-independent inhibition of ERK activation. Oncogene 19: 4531-4541, 2000. 